Revisão / Review

\title{
Tratamento da anemia ferropriva com ferro por via oral
}

Treatment of iron deficiency anemia with oral iron

\author{
Rodolfo D. Cançado ${ }^{I}$ \\ Clarisse Lobo $^{2}$ \\ João Ricardo Friedrich ${ }^{3}$
}

\begin{abstract}
A anemia ferropriva permanece como uma das deficiencias nutricionais mais frequentes e importantes no mundo. O tratamento com ferro deve ser iniciado preferencialmente por via oral e a investigação apropriada de sua causa é obrigatória. Os autores discutem os compostos com ferro atualmente disponiveis, o perfil de eficácia, segurança e tolerabilidade desses medicamentos, e o plano terapêtico mais adequado possivel para o sucesso no tratamento dessa doença tão comum e importante. Rev. Bras. Hematol. Hemoter. 2010;32(Supl.2):114-120.
\end{abstract}

Palavras-chave: Deficiencia de ferro; anemia ferropriva; compostos ferrosos; compostos férricos.

\section{Introdução}

O tratamento da anemia ferropriva foi introduzido por Blaud, em 1832, com um composto cujo principal constituinte era o carbonato férrico. A "pílula de Blaud" permaneceu como pilar do tratamento da defíciência de ferro por mais de cem anos, até o aparecimento de novos compostos com ferro. ${ }^{1}$

O tratamento da anemia ferropriva consiste da orientação nutricional (tema específico abordado em outro artigo deste fascículo), administração por via oral ou parenteral de compostos com ferro e, eventualmente, transfusão de hemácias.

A identificação e correção, quando possível, da causa - ou causas - que levaram à anemia, associadas à reposição do ferro, na dose correta e por tempo adequado, resultam, na maioria das vezes, na sua correção e, consequentemente, confirmam o diagnóstico.

Tratar a anemia ferropriva sem identificar sua causa pode significar a perda da oportunidade de se diagnosticar uma doença subjacente maligna em fase ainda potencialmente curável.

\section{Tratamento com ferro por via oral}

A melhor opção de reposição de ferro é por via oral. O benefício real de um suplemento de ferro está condicionado a fatores como efetividade terapêutica, tolerância gastrointestinal, incidência de eventos adversos, perfil de segurança com risco mínimo de toxicidade e número de tomadas diárias necessárias. Por outro lado, a correção da anemia ferropriva sofre influência de fatores, tais como: intensidade da anemia, capacidade de tolerância e de absorção intestinal do paciente aos suplementos com ferro e presença de doença concomitante. ${ }^{2-4}$

Os principais suplementos de ferro disponíveis e comercializados em diferentes países, inclusive no Brasil, são: sais ferrosos, sais férricos, ferro aminoquelado, complexo de ferro polimaltosado (ferripolimaltose) e ferro carbonila. Em

${ }^{I}$ Médico hematologista. Professor Adjunto da Disciplina de Hematologia e Oncologia da Faculdade de Ciências Médicas da Santa Casa de São Paulo - São Paulo-SP.

${ }^{2}$ Hematologista e hemoterapeuta. Diretora do Hemocentro do Rio de Janeiro - Hemorio - Rio de Janeiro-RJ.

${ }^{3}$ Médico hematologista do Serviço de Hematologia e Transplante de Medula Óssea do Hospital de Clínicas de Porto Alegre-RS.

Faculdade de Ciências Médicas da Santa Casa de São Paulo. Setor de Gestação de Alto Risco do Departamento de Obstetrícia e Ginecologia da Irmandade da Santa Casa de Misericórdia de São Paulo - São Paulo-SP.

Correspondência: Rodolfo Delfini Cançado

Hemocentro da Santa Casa de São Paulo

Rua Marquês de Itú, $579-3^{\circ}$ andar

01223-001 - São Paulo-SP - Brasil

Tel.: (55 11) 2176-7255

E-mail:rdcan@uol.com.br

Doi: $10.1590 /$ S1516-84842010005000062 
função das propriedades farmacológicas e farmacocinéticas dos diferentes compostos, frequência de utilização e importância a nível mundial, enfatizamos neste artigo os resultados de estudos científicos publicados e a nossa experiência com os sais ferrosos e a ferripolimaltose.

\section{Dose de ferro elementar recomendada}

A dose terapêutica de ferro elementar recomendada para o tratamento da anemia ferropriva é de $3 \mathrm{mg}$ a $5 \mathrm{mg} / \mathrm{kg} / \mathrm{dia}$ por um período suficiente para normalizar os valores da hemoglobina $(\mathrm{Hb})$ - de um a dois meses, e restaurar os estoques normais de ferro do organismo - de dois a seis meses, ou até obter-se valor de ferritina sérica de, pelo menos, $15 \mathrm{ng} / \mathrm{mL}$ para crianças e $30 \mathrm{ng} / \mathrm{mL}$ para adultos. Portanto, a duração do tratamento pode variar amplamente, dependendo da intensidade da deficiência de ferro, da sua causa e da dose diária de ferro elementar administrada. ${ }^{3-5}$

Administração de doses diárias superiores a $200 \mathrm{mg}$ não são recomendadas, pois, nesse caso, a mucosa intestinal atua como barreira, impedindo a interiorização e absorção do ferro. ${ }^{5}$

\section{Sais ferrosos}

Os sais ferrosos administrados por via oral são rapidamente absorvidos e bastante eficazes em corrigir hemoglobina e normalizar os estoques de ferro do organismo. Entretanto, o ferro ferroso apresenta algumas desvantagens que acabam limitando o seu uso e efetividade, comprometendo o sucesso terapêutico. ${ }^{6}$

Os sais ferrosos podem interagir com substâncias como fitatos, polifenóis e outros componentes da dieta na luz intestinal, formando complexos insolúveis que impedem a sua absorção. Desta forma, recomenda-se que o ferro ferroso seja ingerido com o estômago vazio, uma hora antes das refeições, entre as refeições ou antes de dormir. Estima-se que a quantidade de ferro absorvida com um comprimido de sulfato ferroso, quando administrado antes da refeição, corresponde à quantidade de ferro absorvida com três comprimidos deste composto quando administrado após a refeição. $2,3,5-7$

$\mathrm{O}$ ferro ferroso pode ser oxidado à $\mathrm{Fe}^{3+}$ na luz intestinal, e esse processo de oxidação na porção apical da membrana enterocítica gera radicais livres capazes de ocasionar a peroxidação lipídica de proteínas da membrana do enterócito e, por conseguinte, provocar dano celular, favorecendo o aparecimento de lesões inflamatórias (esofagite, gastrite, duodenite, úlcera). ${ }^{8}$

A absorção do ferro na sua forma ferrosa $\left(\mathrm{Fe}^{2+}\right)$ dá-se por absorção ativa através da proteína transportadora de metal divante 1 (DMT1) e por difusão passiva na membrana apical do enterócito e intercelular ou paracelular. Uma vez no enterócito, o ferro $\left(\mathrm{Fe}^{2+}\right)$ pode ali ser utilizado pela célula, permanecer sob a forma de ferritina ou dirigir-se à membrana baso-lateral onde, após passar pela ferroportina e ser oxidado à $\mathrm{Fe}^{3+}$, será levado para locais de utilização ou armazenamento pela transferrina. ${ }^{9-11}$

O sulfato ferroso tem a vantagem de poder, em situações de deficiência de ferro moderada a grave, ser absorvido por difusão passiva. Estima-se que até $20 \%$ da quantidade do ferro ferroso não interiorizado por absorção ativa possa ser absorvido por difusão passiva, o que explica o incremento mais rápido da concentração da $\mathrm{Hb}$ observado em alguns estudos quando se utiliza sulfato ferroso em comparação ao incremento da $\mathrm{Hb}$ observado com os sais aminoquelados ou com a ferripolimaltose.

Por outro lado, a possibilidade de absorção ativa e passiva confere ao sal ferroso duas importantes desvantagens. A maior quantidade de ferro absorvida pode ultrapassar a capacidade de saturação da transferrina, ocasionando a presença de ferro não ligado à transferrina (NTBI, nontransferrin-bound iron) e, sobretudo, do ferro livre plasmático (LPI, labile plasma iron), que corresponde à fração do ferro livre mais tóxica para o organismo e maior risco de intoxicação aguda em casos de sobredose do sal ferroso. A administração de doses excessivas do sal ferroso pode superar facilmente a "proteção da mucosa" e causar toxicidade aguda. ${ }^{9-11}$

De fato, o Centro de Controle de Intoxicações dos EUA informou em 1997 que, durante a década de 1986-1996, foram relatados 100 mil casos de intoxicação aguda por compostos com ferro ferroso em crianças menores de 6 anos de idade, chamando a atenção dos perigos do uso indiscriminado destes compostos, considerados e classificados, ainda hoje, como suplementos vitamínicos. ${ }^{12}$

Apesar da eficácia dos compostos com sal ferroso, estes apresentam elevada frequência de efeitos adversos (EA), que pode chegar a $40 \%$ ou mais, e tem relação direta com a quantidade de ferro solúvel presente no trato gastrointestinal superior. Os EA mais frequentemente relatados são: pirose, epigastralgia, náusea, vômito, gosto metálico, escurecimento do esmalte dentário, dispepsia, plenitude ou desconforto abdominal, diarreia e obstipação. O maior obstáculo ao sucesso da terapia com sulfato ferroso é a náusea e desconforto epigástrico, que ocorre 30 a 60 minutos após a ingestão do medicamento. Estes sintomas têm relação direta com a dose administrada e, em alguns casos, podem desaparecer dois a três dias após o início da terapia sem necessidade de descontinuação da mesma. Sintomas como diarreia e obstipação não estão relacionados à dose e podem ser minimizados com tratamento sintomático. ${ }^{3,5,6}$

A quantidade de ferro elementar varia de acordo com o sal ferroso. O sulfato ferroso, fumarato ferroso e gluconato ferroso contêm $20 \%, 33 \%$ e apenas $12 \%$ de ferro elementar, respectivamente. Independentemente do tipo de sal ferroso administrado, a quantidade de ferro absorvida varia entre $<5 \%$ a, no máximo, $50 \%$, e dá-se predominantemente por absorção ativa nos enterócitos duodenais. ${ }^{3,9-11}$ 
A elevada frequência e intensidade dos EA com os sais ferrosos determinam menor tolerância, pior adesão ao tratamento e, consequentemente, piores resultados. Além disso, a dose terapêutica recomendada dos sais ferrosos muitas vezes não é a ideal, mas a tolerada pelo paciente, e esta dose será determinada em função da relação entre benefícios terapêuticos desejados e efeitos tóxicos apresentados.

Apesar dos fatos mencionados anteriormente, o sulfato ferroso é o composto disponível para o tratamento e prevenção da deficiência de ferro em quase a totalidade dos pacientes atendidos pelo Sistema Único de Saúde do Brasil.

\section{Como minimizar os eventos adversos dos sais ferrosos?}

Do ponto de vista prático, recomenda-se adotar algumas medidas com o objetivo de conseguirmos minimizar os EA e melhorar a adesão ao tratamento com os sais ferrosos, tais como: fracionar a dose total diária recomendada em duas ou três tomadas; orientar o paciente para que tome o medicamento durante ou após as refeições, sendo que, nestes casos, a diminuição da quantidade de ferro absorvida poderá ser compensada pelo aumento da adesão ao tratamento e de sua duração; iniciar a terapia com doses menores que a preconizada, ou seja, 40 a $80 \mathrm{mg} / \mathrm{dia}$, aumentando-as gradativamente, de acordo com a tolerância do paciente. $3,5,7,13$

Sabe-se que, quando se administram doses diárias menores que $60 \mathrm{mg}$ de ferro elementar, a frequência e intensidade dos EA observados são similares entre sal ferroso e placebo. Desta forma, vários estudos clínicos utilizaram o sulfato ferroso em diferentes esquemas posológicos, como: $25 \mathrm{mg}$ de ferro elementar uma vez por semana; ${ }^{13} 60 \mathrm{mg}$ de ferro elementar uma vez por dia, duas vezes por semana ou uma vez por dia, diariamente, ${ }^{15} 60 \mathrm{mg}$ de ferro elementar uma vez por semana ou uma vez por dia, diariamente, ${ }^{16} \mathrm{e}$ demonstraram claramente que a adesão ao tratamento é melhor quando se utilizam doses menores em intervalos maiores; entretanto, o sucesso terapêutico não é o esperado, ou o tempo necessário para se atingir o resultado esperado é bem mais demorado (semanas ou meses).

Hallberg et al. ${ }^{6}$ avaliaram os principais EA da terapia com ferro por via oral estudando indivíduos com anemia ferropênica e comparando diferentes grupos tratados com sulfato ferroso, fumarato ferroso, gluconato ferroso e placebo. Quando se comparou grupo com sulfato ferroso ( $\mathrm{n}=198)$ com dose diária de $222 \mathrm{mg}$ de ferro elementar $(37 \mathrm{mg}$ $3 \mathrm{x} /$ dia) versus placebo $(\mathrm{n}=195)$, observaram-se $22,6 \%$ e $13,6 \%$ de EA, respectivamente; e $8 \%$ e $4,1 \%$ de descontinuação do tratamento, respectivamente, com diferença estatisticamente significante entre os grupos estudados.

Quando se comparou a administração da dose diária de $222 \mathrm{mg}$ de ferro elementar ( $37 \mathrm{mg} \mathrm{3x} /$ dia) de sulfato ferroso $(\mathrm{n}=120)$, fumarato ferroso $(\mathrm{n}=118)$ e gluconato ferroso $(\mathrm{n}=120)$ versus placebo $(\mathrm{n}=119)$, observaram-se $27,9 \%, 26,4 \%$ e $31,5 \%$ versus $13,9 \%$ de EA, respectivamente; e $9,9 \%, 5,5 \%$ e $5,4 \%$ versus $0,9 \%$ de descontinuação do tratamento, respectivamente, diferenças estas estatisticamente significantes entre os grupos com sal ferroso e placebo. ${ }^{6}$

\section{Ferro aminoquelado}

Os sais aminoquelados resultam da união covalente do ferro em sua forma ferrosa $\left(\mathrm{Fe}^{2+}\right)$ ou férrica $\left(\mathrm{Fe}^{3+}\right)$ a um ligante orgânico. A molécula resultante é um metal quelado, que tem como finalidade apresentar uniões de quelação suficientemente fortes para resistir à ação de enzimas e proteínas da digestão, e de substâncias naturais presentes nos alimentos, como fitato, folato, ácido tânico. Além disso, protege os átomos de ferro, reduzindo a exposição direta das células da mucosa gastrointestinal a este metal, o que poderia reduzir a toxicidade local. ${ }^{17}$

Os ligantes orgânicos mais frequentemente utilizados são os aminoácidos, pelo fato destes serem captados predominantemente no jejuno, onde estas proteínas são hidrolizadas e absorvidas.

Embora tenham sido formulados dezenas de compostos aminoquelados, os mais estudados são o bisglicinato (que contém $20 \%$ de ferro elementar), o trisglicinato férrico e a glicina-sulfato ferroso. ${ }^{17}$

Os compostos aminoquelados teoricamente têm menor incidência de efeitos secundários descritos em relação às apresentações ferrosas não queladas; entretanto, são menos eficientes que os complexos de ferro polimaltosado $\left(\mathrm{Fe}^{3+}\right)$, sobretudo com relação à menor incidência de EA gastrointestinais observada com estes últimos.

Além disso, a biodisponibilidade dos sais aminoquelados é bastante variável e inferior à constatada em vários outros compostos, razão pela qual a agência FDA norteamericana tem recomendado este tipo de sal para a fortificação de alimentos, mas não como suplemento terapêutico.

Hallberg et al. ${ }^{6}$ compararam a administração da dose diária de $180 \mathrm{mg}$ de ferro elementar (60 mg 3x/dia) de sulfato ferroso $(n=195)$, ferro aminoquelado (sulfato ferroso-glicina, $\mathrm{n}=200)$ e fumarato ferroso $(\mathrm{n}=196)$ versus placebo $(\mathrm{n}=200)$, e observaram $26,5 \%, 24,4 \%$ e $27,0 \%$ versus $12,4 \%$ de EA, respectivamente, não havendo diferença estatisticamente significante entre os grupos que receberam sal ferroso, embora esta diferença tenha sido significante entre grupos com sal ferroso e placebo.

Este estudo mostra que os diferentes tipos de sal ferroso apresentam incidência e tipo de EA semelhantes entre si quando se utiliza a mesma dose de ferro elementar de cada sal.

Coplin et al. ${ }^{17}$ comparando tolerabilidade entre sulfato ferroso e ferro aminoquelado (bis-glicinato) mostraram eficácia terapêutica semelhante em doses equivalentes $(50 \mathrm{mg}$ de ferro elementar), porém a frequência de EA foi de $37 \%$ no grupo com sulfato ferroso e de $21 \%$ no grupo com o ferro aminoquelado. 


\section{Complexo de ferro polimaltosado $\left(\mathrm{Fe}^{3+}\right)$}

$\mathrm{O}$ hidróxido de ferro férrico $\left(\mathrm{Fe}^{3+}\right)$ polimaltosado não iônico, também conhecido como ferripolimaltose, é um complexo hidrossolúvel, constituído de hidróxido de ferro férrico polinuclear e dextrina parcialmente hidrolizada (polimaltose). A superfície dos núcleos de hidróxido de ferro $\left(\mathrm{Fe}^{3+}\right)$ polinuclear está rodeada por várias moléculas de polimaltose ligadas por união covalente, o que resulta em um complexo de PM de aproximadamente $52.300 \mathrm{Da}^{18}$

Jacobs et al. ${ }^{18}$ demonstraram que a biodisponibilidade do ferro nas formulação de sulfato ferroso ou ferripolimaltose são semelhantes tanto quando se utilizaram doses "fisiológicas" de $5 \mathrm{mg}$ dos dois compostos como doses terapêuticas diárias de $50 \mathrm{mg}$ de ferro elementar. A taxa média de absorção para ambos os sais foi de $50 \%$ para a dose de $5 \mathrm{mg}$ e de $30 \%$ para $50 \mathrm{mg}$. Constatou-se também correlação inversa entre taxa de absorção e valor de ferritina plasmática, ou seja, quanto menor a ferritina, maior a absorção do ferro administrado por via oral. Esta bioequivalência também foi constatada entre ferripolimaltose e fumarato ferroso.

Acredita-se que a ferripolimaltose liga-se às moléculas de DMT1 na superfície apical dos enterócitos, predominantemente duodenais, e que haja um intercâmbio competitivo de ligantes - denominada absorção fisioativa ou físiologicamente controlada, ou seja, o ferro férrico desliga-se da polimaltose e, após ser reduzido a $\mathrm{Fe}^{2+}$ na membrana apical, ligase a receptores da proteína DMT1 através da qual o ferro $\mathrm{Fe}^{2+}$ chega ao citoplasma e poderá ali permanecer sob a forma de ferritina ou dirigir-se à membrana baso-lateral onde, após passar pela ferroportina e ser oxidado à $\mathrm{Fe}^{3+}$, será levado para locais de utilização ou armazenamento pela transferrina. ${ }^{9-11,18}$

A ferripolimaltose é uma molécula grande, o que explica o fato de que sua difusão passiva através da membrana da mucosa intestinal é cerca de quarenta vezes menor que a observada com o ferro ferroso não quelado. Isto justifica o incremento mais lento da $\mathrm{Hb}$ no início do tratamento quando comparado ao incremento obtido com o sal ferroso; entretanto, o risco de toxicidade aguda com a ferripolimaltose é cerca de dez vezes menor que o observado com o sulfato ferroso. A dose média letal da ferripolimaltose em animais experimentais, quando administrado por via oral, é maior que $2.000 \mathrm{mg} / \mathrm{kg}$ de peso corporal, enquanto esta dose é de $350 \mathrm{mg} / \mathrm{kg}$ com o sulfato ferroso. ${ }^{11,18,20}$

Portanto, diferentemente dos sais ferrosos, a ferripolimaltose não é absorvida por difusão passiva apical ou paracelular, não sofre processo de oxidação e não ocasiona a presença de ferro livre plasmático. Outra vantagem da ferripolimaltose em rela- ção aos sais ferrosos é a de que este complexo pode ser administrado durante ou após a refeição, pois sua absorção não sofre influência dos alimentos. ${ }^{9-11,18}$

As propriedades mencionadas anteriormente conferem à ferripolimaltose menor incidência de EA, proporcionando maior adesão ao tratamento e melhores resultados. ${ }^{17-19}$

Estudos em animais têm demonstrado que o processo de oxidação necessário para a absorção dos sais ferrosos provoca geração de radicais livres e lesão celular, o que pode justificar o maior incremento dos níveis da ferritina nos pacientes tratados com sulfato ferroso. Entretanto, quando se analisa o incremento de ferritina tecidual, que é o que realmente interessa, esse aumento é maior para aqueles que recebem a ferripolimaltose que para os que recebem o sulfato ferroso ${ }^{8,21}$ Além disso, o risco de toxicidade hepática é menor com a ferripolimaltose que com os sais ferrosos. ${ }^{22}$

As principais características farmacológicas e diferenças entre sais ferrosos e a ferripolimaltose estão relacionadas no Quadro 1.

\section{Ferripolimaltose versus sais ferrosos ou aminoquelados}

Vários estudos têm demonstrado que a ferripolimaltose confere excelente resultado quanto à correção da anemia e o reestabelecimento dos estoques normais de ferro do organismo em lactentes, crianças e adultos. ${ }^{18,21,23,24}$

Borbolla $\mathrm{Jr}$ et al. compararam a administração de ferripolimaltose $(\mathrm{n}=15)$ versus sulfato ferroso $(\mathrm{n}=15)$ no tratamento da anemia por deficiência de ferro em lactentes. Os autores observaram eficácia clínica e laboratorial, e incidência de EA similares entre ambos os grupos. ${ }^{24}$

Jacobs et al. ${ }^{20}$ comparando a eficácia e segurança do uso de sulfato ferroso ( $100 \mathrm{mg} 2 \mathrm{x} /$ dia por 12 semanas, $\mathrm{n}=47$ ) e da ferripolimaltose ( $200 \mathrm{mg}$ por 12 semanas, $\mathrm{n}=46$ ) no trata-

Quadro 1. Principais características farmacológicas e diferenças entre sais ferrosos e a ferripolimaltose

\begin{tabular}{|c|c|c|}
\hline Parâmetro & Sais ferrosos & Ferripolimaltose \\
\hline Absorção & $\begin{array}{c}\text { Mais rápida, } \\
\text { difusão ativa e passiva } \\
\text { não controlada }\end{array}$ & $\begin{array}{c}\text { Mais lenta, } \\
\text { difusão ativa } \\
\text { fisiologicamente controlada }\end{array}$ \\
\hline Administração & Jejum & $\begin{array}{l}\text { Durante ou } \\
\text { após a refeição }\end{array}$ \\
\hline Eficácia & Elevada & Elevada \\
\hline $\begin{array}{l}\text { Frequência de } \\
\text { eventos adversos }\end{array}$ & $\begin{array}{c}\text { Elevada } \\
(5 \% \text { a } 45 \%)\end{array}$ & $\begin{array}{c}\text { Baixa } \\
(0 \% \text { a } 15 \%)\end{array}$ \\
\hline $\begin{array}{l}\text { Estresse oxidativo nas } \\
\text { células da mucosa do trato } \\
\text { gastrointestinal }\end{array}$ & Presente & Ausente \\
\hline Mancha nos dentes & Sim & Não \\
\hline $\begin{array}{c}\text { Tolerância e } \\
\text { adesão ao tratamento }\end{array}$ & Menor & Maior \\
\hline
\end{tabular}


mento de doadores de sangue com anemia ferropriva, constataram aumento similar da $\mathrm{Hb}$ para os dois grupos e maior aumento da ferritina sérica com o sulfato ferroso. Em relação à incidência de EA, observaram-se taxas de $44,7 \%$ com sulfato ferroso e de $17,5 \%$ com ferripolimaltose; e quanto à tolerância, observou-se taxa maior que $80 \%$ com a ferripolimaltose e de $60 \%$ com o sulfato ferroso. Dos pacientes que descontinuaram o tratamento, a principal causa foi náusea e ocorreu em $3 \%$ daqueles com ferripolimaltose e $19 \%$ dos pacientes recebendo sulfato ferroso.

O estudo prospectivo controlado e randomizado mais importante é o de Jacobs et al.,${ }^{19}$ que compararam a biodisponibilidade e bioequivalência da ferripolimaltose e do sulfato ferroso em candidatos à doação de sangue e recusados para tal procedimento devido ao diagnóstico de anemia ferropriva. Os autores utilizaram $120 \mathrm{mg}$ de sulfato ferroso (60 mg 2x/dia, em jejum; $\mathrm{n}=51$ ) versus $100 \mathrm{mg}$ de ferripolimaltose no café da manhã $(\mathrm{n}=55)$ versus $200 \mathrm{mg}$ de ferripolimaltose ( $100 \mathrm{mg}$ no café e no jantar; $\mathrm{n}=53$ ). Os três grupos apresentaram aumento satisfatório da $\mathrm{Hb}$ após quatro semanas de tratamento e restabelecimento dos estoques de ferro após 12 semanas de tratamento. A incidência de náusea e vômitos foi significativamente maior para o grupo com sulfato ferroso do que para os grupos com ferripolimaltose; dez pacientes com sulfato ferroso descontinuaram o tratamento por EA, enquanto este fato não ocorreu em nenhum paciente com ferripolimaltose. Não houve diferença significativa de ocorrência de EA entre os grupos com $100 \mathrm{mg}$ e $200 \mathrm{mg}$ de ferripolimaltose, entretanto, o incremento de $\mathrm{Hb}$ e da ferritina foi menor para o grupo com $100 \mathrm{mg} /$ dia.

Este é o primeiro estudo que demonstrou que uma dose diária de $200 \mathrm{mg}$ de ferripolimaltose ( $100 \mathrm{mg} 2 \mathrm{x}$ ao dia) é mais efetiva que uma dose de $100 \mathrm{mg}$, sem incremento da taxa de EA; que $200 \mathrm{mg}$ de ferripolimaltose equivalem a $120 \mathrm{mg}$ de sulfato ferroso, com a grande vantagem a favor da ferripolimaltose em função da menor incidência de EA e descontinuação do tratamento, o que torna este produto como medicamento de escolha no tratamento da anemia ferropênica, pelo menos aos indivíduos intolerantes ao sulfato ferroso.

Pacientes idosos apresentam maior incidência de EA quando tratados com ferro por via oral na dose terapêutica recomendada. Nestes casos, doses inferiores, até mesmo de $15 \mathrm{mg}$ de ferro elementar por dia, podem ser efetivas nos casos de anemia leve devido à menor ocorrência de EA. ${ }^{13}$

Madero $^{25}$ avaliou a eficácia e segurança da ferripolimaltose $(n=36)$ e do ferro aminoquelado $(n=33)$ no tratamento da anemia ferropênica em crianças com idade variando entre 6 e 14 meses. Este estudo observou que houve aumento da $\mathrm{Hb}$ similar e sem diferença estatisticamente significativa nos dois grupos, embora a taxa de incremento tenha sido maior nos pacientes tratados com a ferripolimaltose. A porcentagem de pacientes que obtiveram aumento da $\mathrm{Hb}$ maior que $1,5 \mathrm{~g} / \mathrm{dL}$ no dia 30 de tratamento foi de $77,6 \%$ e $63,6 \%$ com a ferripolimaltose e ferro aminoquelado, respectivamen- te. A ocorrência de EA foi significativamente maior com o ferro aminoquelato que com a ferripolimaltose $(33,3 \%$ e $13,8 \%$, respectivamente; $\mathrm{p}=0,05$ ).

Recente meta-análise, ao comparar o uso de sulfato ferroso ( $\mathrm{n}=238)$ e o de ferripolimaltose $(\mathrm{n}=319)$ em pacientes adultos com anemia ferropriva, mostrou que os dois compostos apresentam eficácia semelhante em relação ao aumento da concentração da $\mathrm{Hb}$ circulante; entretanto, houve maior ocorrência de EA e menor tolerância no grupo dos que foram tratados com sulfato ferroso, e o tratamento com a ferripolimaltose obteve melhores resultados com relação à reposição dos estoques de ferro. ${ }^{26} \mathrm{O}$ Quadro 2 descreve a frequência de eventos adversos relatados por pacientes com anemia ferropriva tratados com sulfato ferroso ou ferripolimaltose.

Quadro 2. Frequência de eventos adversos relatados de acordo com o composto de ferro utilizado ${ }^{26}$

\begin{tabular}{cccc}
\hline Evento adverso & $\begin{array}{c}\text { Sulfato ferroso } \\
\mathrm{n}=238\end{array}$ & $\begin{array}{c}\text { Ferripolimaltose } \\
\mathrm{n}=319\end{array}$ & $\mathrm{p}$ \\
\hline $\begin{array}{c}\text { Náusea, pirose, } \\
\text { epigastralgia }\end{array}$ & $45,7 \%$ & $13,1 \%$ & $<0,001$ \\
Diarreia & $15,4 \%$ & $5,9 \%$ & $<0,001$ \\
Constipação & $15,9 \%$ & $8,7 \%$ & $<0,05$ \\
Mancha nos dentes & $7,7 \%$ & $0,7 \%$ & $<0,001$ \\
\hline
\end{tabular}

Propriedades da ferripolimaltose, como mecanismo de absorção intestinal fisiologicamente controlado, excelente biodisponibilidade, frequência de efeitos adversos e risco de toxicidade significativamente inferiores aos observados com outras formas de suplementos de ferro por via oral conferemlhe e asseguram-lhe excelente resposta terapêutica e tornam este composto uma opção de grande valia.

Entretanto, vale a pena lembrar e destacar que o alto grau de tecnologia necessário para a produção de compostos como a ferripolimaltose leva-nos a duvidar ou, pelo menos, a questionar, quanto à bioequivalência, perfil de eficácia e segurança de compostos diferentes do original comercializados por diferentes fabricantes em diferentes países.

\section{Quantidade de ferro elementar}

É importante que o médico tenha ciência da quantidade exata de ferro elementar do composto que está prescrevendo para o tratamento de seu paciente, pois esta varia consideravelmente de acordo com o medicamento utilizado ou disponível. Os principais sais de ferro disponíveis para suplementação por via oral estão relacionados no Quadro 3.

A primeira fase do tratamento da anemia ferropriva corresponde à normalização dos valores da $\mathrm{Hb}$, e o tempo necessário para essa correção é de um a dois meses, ou mais, dependendo da intensidade da anemia, tolerância ao tratamento e correção da causa que levou à deficiência de ferro. 
Quadro 3. Principais compostos com ferro disponíveis para tratamento da anemia ferropriva por via oral

\begin{tabular}{|c|c|c|c|}
\hline $\begin{array}{l}\text { Composto } \\
\text { com ferro }\end{array}$ & $\begin{array}{l}\text { Peso em mg } \\
\text { do sal de ferro } \\
\text { por comprimido }\end{array}$ & $\begin{array}{l}\text { Quantidade em mg } \\
\text { de ferro elementar } \\
\text { por comprimido }\end{array}$ & Observação \\
\hline Ferripolimaltose & 333 & 100 & $\begin{array}{l}1 \mathrm{~mL} \text { (20 gotas) contém } \\
50 \mathrm{mg} \text { de ferro elementar; } \\
10 \mathrm{~mL} \text { do xarope contém } \\
100 \mathrm{mg} \text { de ferro elementar }\end{array}$ \\
\hline Sulfato ferroso & 300 & 40 a 60 & $\begin{array}{c}1 \mathrm{~mL} \text { (20 gotas) contém } \\
25 \mathrm{mg} \text { de ferro elementar; } \\
10 \mathrm{mLdo} \text { xarope contém } \\
80 \mathrm{mg} \text { de ferro elementar }\end{array}$ \\
\hline Fumarato ferroso & 200 & 30 a 60 & - \\
\hline Gluconato ferroso & 300 & 36 & - \\
\hline $\begin{array}{l}\text { Ferro quelato } \\
\text { glicinato }\end{array}$ & 150 a 300 & 30 a 100 & $\begin{array}{l}\text { Flaconete de } 5 \mathrm{~mL} \text { com } \\
50 \mathrm{mg} \text { de ferro elementar }\end{array}$ \\
\hline
\end{tabular}

Quadro 4. Causas mais frequentes de falha no tratamento com ferro por via oral

Continuidade da perda de sangue por falha na identificação ou correção de sangramento e/ou de distúrbio de absorção de ferro

Medicação usada inadequadamente - baixa adesão ao tratamento devido aos EA gastrointestinais e/ou dose inadequada e/ou duração insuficiente

Doença coexistente interferindo na resposta ao tratamento com ferro por via oral doença renal crônica; doença inflamatória ou infecciosa associada

Doenças associadas com distúrbio de absorção do ferro - doença celíaca, gastrite atrófica autoimune e infecção pelo helicobacter pylori

Diagnóstico incorreto

Interação medicamentosa

Deficiências nutricionais combinadas
Causas genéticas de deficiência de ferro

repetida a cada trinta a sessenta dias, até a obtenção de valores de ferritina de pelo menos $15 \mathrm{ng} / \mathrm{mL}$ para crianças e $30 \mathrm{ng} / \mathrm{mL}$ para adultos, o que indica a reconstituição das reservas normais de ferro e sinaliza a suspensão do tratamento. . $^{3,4,5,7}$

\section{Causas de falha do tratamento}

As causas mais frequentes de falha no tratamento com ferro por via oral, ou de sua refratariedade, estão relacionadas no Quadro 4. 3,4,5,7

A reposição com ferro por via oral é eficaz no tratamento da maioria dos pacientes com anemia ferropênica, entretanto, em algumas situações específicas, a terapia por via oral é insuficiente para normalizar a $\mathrm{Hb}$ e/ou reestabelecer os depósitos normais de ferro. Nestes casos, a administração de ferro por via parenteral é uma alternativa eficaz, efetiva e segura, e deve ser considerada (tema específico abordado em outro artigo deste fascículo).

\section{Conclusões}

- Toda situação de deficiência de ferro tem causa e sua investigação é imprescindível

- A anemia ferropriva continua sendo um grave problema de Saúde Pública, principalmente em países em desenvolvimento

Os principais critérios de boa resposta ao tratamento com ferro por via oral são: redução ou desaparecimento de sintomas e sinais relacionados à deficiência de ferro (cansaço, queda de cabelo, etc); maior tolerância à atividade física; reticulocitose, que já pode ser observada entre o $3^{\circ}$ e o $5^{\circ}$ dia do início do tratamento e é máxima entre o $8^{\circ}$ e $10^{\circ}$ dia; e aumento do valor da $\mathrm{Hb}$ de, pelo menos, $2 \mathrm{~g} / \mathrm{dL}$ após duas a três semanas de tratamento. ${ }^{2-4}$

A segunda fase do tratamento corresponde à reposição dos estoques de ferro do organismo. Para esta fase, a duração do tratamento pode variar de dois a seis meses, e o sucesso terapêutico desta fase está relacionado, principalmente, à tolerância do paciente ao composto com ferro que está fazendo uso.

Com relação ao controle laboratorial do paciente em tratamento com ferro por via oral, preconiza-se a dosagem da hemoglobina a cada duas a quatro semanas de tratamento. Trinta a 45 dias após a correção da anemia, ou seja, após obtido o valor de $\mathrm{Hb}$ de, pelo menos, $12 \mathrm{~g} / \mathrm{dL}$ para as mulheres, $11 \mathrm{~g} / \mathrm{dL}$ para as gestantes e de $13 \mathrm{~g} / \mathrm{dL}$ para os homens, recomenda-se a dosagem de ferritina sérica, que pode ser
- A melhor via para a reposição de ferro é a oral, e a dose terapêutica recomendada é de $3 \mathrm{mg}$ a $5 \mathrm{mg} / \mathrm{kg} / \mathrm{dia}$, por período suficiente para normalizar os valores da hemoglobina (de um a dois meses) e restaurar os estoques normais de ferro do organismo (de dois a seis meses, ou obtenção de ferritina sérica $>15 \mathrm{ng} / \mathrm{mL}$ para crianças e $>30 \mathrm{ng} / \mathrm{mL}$ para adultos). Portanto, são necessários pelo menos noventa dias de tratamento.

- A adequada orientação dos pacientes quanto aos possíveis EA, a importância da adesão e a duração do tratamento são fundamentais para o sucesso terapêutico.

- A quantidade de ferro elementar de cada composto com ferro varia amplamente e esse conhecimento por parte do médico prescritor é fundamental para o sucesso do tratamento.

- Via de regra, todos os compostos com ferro (sais ferrosos, sais aminoquelados e a ferripolimaltose) apresentam eficácia semelhante quanto à correção da $\mathrm{Hb}$; entretanto, a menor ocorrência de EA e maior tolerância documentada com a ferripolimaltose demonstram que este complexo confere melhores resultados em relação aos demais sais de ferro no tratamento completo da anemia ferropriva. 


\section{Abstract}

Iron deficiency anemia remains one of the commonest and most important nutritional deficiencies in the world today. The treatment of iron deficiency should preferably be initiated with oral iron; an appropriate investigation of the cause of iron deficiency anemia is mandatory. The authors discuss the currently available iron preparations as well as their efficacy, safety and tolerability and the optimal therapeutic plan to successfully treat this common and important disease. Rev. Bras. Hematol. Hemoter. 2010;32(Supl.2): 114-120.

Key words: Iron deficiency; anemia, iron-deficiency; ferrous compounds; ferric compounds.

\section{Referências Bibliográficas}

1. Crosby WH. The rationale for treating iron deficiency anemia. Arch Intern Med 1984;144(3):471-2.

2. Baynes RD. Iron deficiency. In: Brock JH, Halliday JW, Pippard MJ, Powell LW. Iron metabolism in health disease. London, W.B. Saunders, 1994. p.189-225.

3. Cook JD. Newer aspects of the diagnosis and treatment of iron deficiency. Hematology Am Soc Hematol Educ Program, 2003; 53-61.

4. Beutler E. Disorders of iron metabolism. In: Williams Hematology. Chapter 40. Seventh Edition. McGraw-Hill, 2006;511-553.

5. Alleyne M, Horne MK, Miller JL. Individualized treatment for iron-deficiency anemia in adults. Am J Med. 2008;121(11):943-8.

6. Cook JD. Diagnosis and management of iron-deficiency anaemia. Best Pract Res Clin Haematol. 2005;18(2):319-32.

7. Hallberg L, Ryttinger L, Solvell L. Side-effects of oral iron therapy. A double-blind study of different iron compounds in tablet form Acta Med Scand Suppl. 1966; 459:3-10.

8. Toblli JE, Cao G, Olivieri L, Angerosa M. Comparative study of gastrointestinal tract and liver toxicity of ferrous sulfate, iron amino chelate and iron polymaltose complex in normal rats. Pharmacology. 2008;82(2):127-37.

9. Jacobs P, Wormald LA, Gregory MC. Absorption of iron polymaltose and ferrous sulphate in rats and humans, a comparative study. S Afr Med J. 1979;55(26):1065-72.

10. Jacobs P, Johnson G, Wood L. Oral iron therapy in human subjects. Comparative absorption between ferrous salts and iron polymaltose. J Med. 1985;15(5-6):367-77.

11. Johnson G, Jacobs P. Bioavailability and the mechanisms of intestinal absorption of iron from ferrous ascorbate and ferric polymaltose in experimental animals. Exp Hematol. 1990; 18(10):1064-9.

12. Faich F, Strobos J. Sodium ferric gluconate complex in sucrose: safer intravenous iron therapy than iron dextrans. Am J Kidney Dis. 1999;33(3):464-70.

13. Rimon E, Kagansky N, Kagansky M, Mechnick L, Mashiah T, Namir M, et al. Are we giving too much iron? Low-dose iron therapy is effective in octogenarians. Am J Med. 2005;118(10): 1142-47.

14. Coutinho GG, Goloni-Bertollo EM, Pavarino-Bertelli EC. Effectiveness of two programs of intermittent ferrous supplementation for treating iron-deficiency anemia in infants: randomized clinical trial. Sao Paulo Med J. 2008;126(6):31-48.
15. Souza AI, Batista Filho M, Bresani CC, Ferreira CCL, Figueiroa JN. Adherence and side effects of three ferrous sulfate treatment regimens on anemic pregnant women in clinical trials. Cad. Saúde Pública, 2009;25(6):1225-33.

16. Lopes MCS, Ferreira LOC, Batista FM. Uso diário e semanal de sulfato ferroso no tratamento de anemia em mulheres no período reprodutivo. Cad. Saúde Pública. 1999;15:799-808.

17. Coplin M, Schuette S, Leichtmann G, Lashner B. Tolerability of iron: a comparison of bis-glycino iron II and ferrous sulfate. Clin Ther. 1991;13(5):606-12.

18. Geisser P. Safety and efficacy of iron(III)-hydroxide polymaltose complex. A review of over 25 years experience. Arzneimittelforschung. 2007;57(6A):439-52.

19. Jacobs P, Fransman D, Coghlan P. Comparative bioavailability of ferric polymaltose and ferrous sulphate in iron-deficient blood donors. J Clin Apher. 1993;8(2):89-955.

20. Jacobs P, Wood L, Bird A. Erythrocytes: better tolerance of iron polymaltose complex compared with ferrous sulphate in the treatment of anaemia. Hematology. 2000;5(1):77-83.

21. Kavakli K, Yilmaz D, Cetinkaya B, Balkan C, Sözmen EY, Sagin FG. Safety profiles of $\mathrm{Fe} 2+$ and $\mathrm{Fe} 3+$ oral preparations in the treatment of iron defiency anemia in children. Pediatr Hematol Oncol. 2004;21(5):403-10.

22. Sas G, Nemesanszky E, Bräuer H, Scheffer K. On the therapeutic effects of trivalent and divalent iron in iron deficiency anaemia. Arzneimittelforschung. 1984;34(11):1575-9.

23. Murahovschi J, Stein ML, Careffa RC, De Andrade VL, Guerra CC, Falci M. Tratamento da ferropenia e da anemia ferropriva com o complexo de hidróxido de ferro polimaltosado por via oral, de crianças em fase de recuperação de infecções respiratórias. Ensaio duplo-cego, comparativo com sulfato ferroso. Rev Paul Ped. 1987; 5:97-104

24. Borbolla JR, Cicero RE, Dibildox MM, Sotres RD. Complejo polimaltosado férrico vs sulfato ferroso em el tratamiento de la anemia por deficiência de hierro em lactantes. Rev Mex Pediatr, 2000;67:63-67.

25. Madero D. Eficacia y seguridad del complejo de hidroxido de hierro férrico (III) y polimaltosa (IPC) vs el hierro aminoquelado em el manejo de la anemia ferropénica em ninos. Rev AWGLA, 2007; $1: 28-39$.

26. Toblli JE, Brignolli R. Iron(III)-hydroxide polymaltose complex in iron deficiency anemia / review and meta-analysis. Arzneimittelforschung. 2007;57(6A):431-8.

O tema foi sugerido e avaliado pelo coeditor deste fascículo educativo, Rodolfo Delfini Cançado, e pelo board interno da RBHH, e publicado após a concordância do editor, Milton Artur Ruiz.

Conflito de interesse: sem conflito de interesse

Recebido: 08/01/2010

Aceito: $01 / 02 / 2010$ 